\title{
Experience Level Agreements in Wireless Metropolitan Area Sharing Networks
}

\author{
Mónica Alejandra Lora Girón \\ Chair of Communication and Distributed Systems \\ RWTH Aachen University \\ Aachen, Germany \\ Monica.Lora@comsys.rwth-aachen.de
}

\begin{abstract}
Nowadays, mobile wireless users are in constant demand of connectivity.

Satisfying the connectivity requirements of the users requires a large amount of Access Points (APs) in order to enlarge the wireless service coverage. Wireless Metropolitan Area Sharing Networks (WMSN) are a low cost alternative that fulfill this high degree of user requirements.

On WMSN certain places will experiment congestion when a large number of users are associated with the same device. For this reason, new mechanisms are required with the aim of satisfy the Quality of User Experience (QoE).

The Experience Level Agreements (ELA) definition for WMSN is a formal way to characterize the requirements of the users and permits having measuring functions for this kind of network, which helps to improve the QoE.
\end{abstract}

\section{Categories and Subject Descriptors}

C. 2 [Computer Systems Organization]: ComputerCommunication Networks

; C.2.1 [Computer-Communication Networks]: Network architecture and Design Wireless Communication

\section{General Terms}

Cooperative Wireless Networks, User Experience

\section{Keywords}

QoE, ELA, IEEE 802.11e, Game theory, WMSN

\section{INTRODUCTION}

The extensive use of wireless connectivity and the increasing demand of services by the users have triggered

Permission to make digital or hard copies of all or part of this work for personal or classroom use is granted without fee provided that copies are not made or distributed for profit or commercial advantage and that copies bear this notice and the full citation on the first page. To copy otherwise, to republish, to post on servers or to redistribute to lists, requires prior specific permission and/or a fee.

ACM CoNEXT 2010, November 30-December 3, 2010, Philadelphia, USA.

Copyright 2010 ACM 978-1-4503-0468-9/10/0011 ...\$10.00.

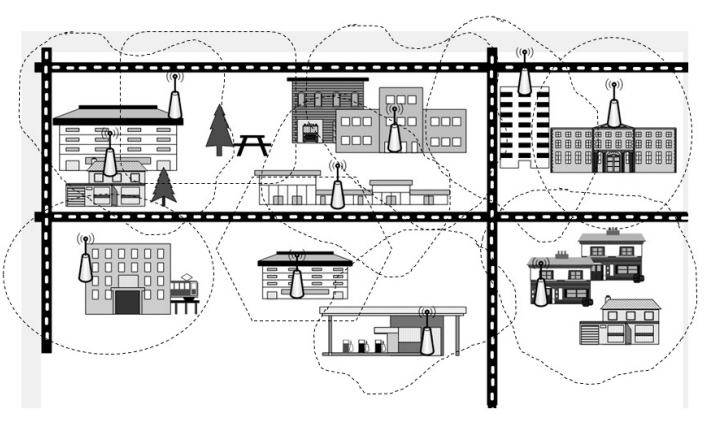

Figure 1: Wireless Metropolitan Area Sharing Network

increasing requirements for performance and coverage in wireless networks. WMSN appear as a solution that ensures the metropolitan coverage without the installation of a large number of Access Points (APs) by one entity, thus saving the cost associated with an infrastructure of this magnitude. A WMSN corresponds to a set of public or/and privately owned APs shared by the visitors and citizens of a city. An example of a WMSN is depicted in Fig.1.

A WMSN involves APs and users with different operational capabilities and load. A major challenge of such a scenario is to guarantee the satisfaction of the users when they access the Internet to use available services, and to balance the load between the network components taking into account the dynamic conditions of this kind of network.

The IEEE defines the IEEE 802.11e Standard [1] for Quality of Service (QoS) support. However, this standard was not designed for the satisfaction of the user experience when the user is accessing the services, i.e. the assessment of the perceptual and application performance.

The ITU-T defines QoE [2] as: "The overall acceptability of an application or service, as perceived subjectively by the end-user."

In order to fulfill this acceptability from the user point of view, we have take into consideration the environmental context and the expectations that the user has. 


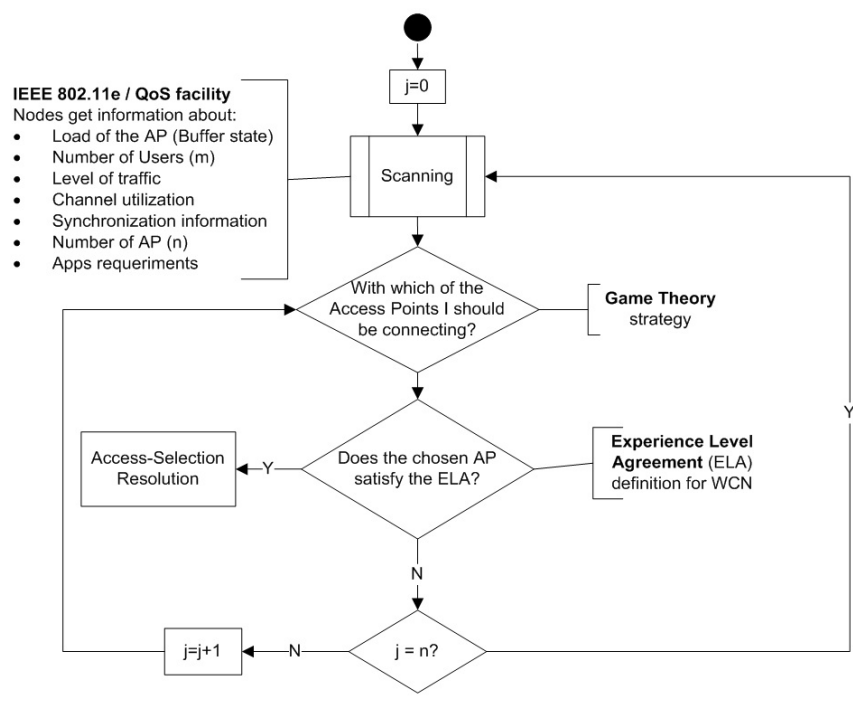

Figure 2: Components flow of the proposal

The remainder of this document is concerned with the design of a new architecture for QoE in a WMSN scenario. The architecture enables the balanced and fair association between the users and the shared APs, and this objective is reached through an AP selection mechanism based on an algorithm that combines the IEEE 802.11e standard with game theory.

\section{PROBLEM STATEMENT}

Typically in 802.11 networks the association process depends on the Received Signal Strength Indicator(RSSI), where the strongest signal corresponds to the AP that the station should be associated with. However, this decision does not consider the load and available capacity at the AP. The APs located in popular and public spaces that suffer from the overloaded bandwidth utilization in that Basic Service Set (BSS) or coverage area will offer low throughput to the clients. Consequently, it is desirable to make a careful selection of the AP, in a way that allows the user to experience the maximum performance and to ensure an efficient balancing of the traffic loads among the APs.

The idea is to guarantee the users of a WMSN the best possible association with the APs, according to their ELA[3]. In turn, these users-competitors can share the bandwidth in the assigned AP according to QoS requirements. The system model is presented in Fig.2.

Our proposal has three different main components:

Firstly, we formulate the problem of finding the subset of preferred APs. The Qos facilities defined in the IEEE 802.11e Standard is employed to solve this problem.

In the second front, we formulate the AP selection problem using a theory game approach. To ensure a QoE means to carry out an optimal allocation of the network resources according to the current requirements of the user. Therefore, we aim to determine the user behavior and to define the best strategy to be applied for those users.

Finally, in the third front we formulate the ELA problem. The access-selection resolution then will be decided according to the satisfaction of this ELA.

Note that we do not consider any centralized entity in the proposed scheme.

\subsection{IEEE 802.11e Standard}

Before a station is associated with an AP, the station gets some synchronization information from the APs in order to choose the AP that has the strongest RSSI. This process is called the scanning phase. During this first exchange of information, the IEEE 802.11e standard provides more elements related to the available admission capacity, current population and traffic levels in the APs. We are using this data to select the set of APs that satisfy the application requirements that the user has.

\subsection{Game Theoretical Approach}

After the scanning phase, the user has a list of the APs that meet the expectations that the user applications have, but users may behave selfishly, seeking to select the AP with less workload, higher performance and better coverage. For this reason we use a game theoretic approach for solving this problem between the user-players in a competitive WMSN environment. In seeking to resolve this conflict, the users can choose the right strategy for them, which corresponds to the AP that provides the proper service in accordance with the QoE estimation. A game model is appropiate because the strategy of each user to impact the outcomes to other users.

\section{CONCLUSIONS}

We have proposed a way to exploit the IEEE 802.11e standard to provide a fair and balanced access for the users in a WMSN. The described work is in progress, and has as goal the testing of this approach as a part of a real WMSN.

\section{REFERENCES}

[1] IEEE 802.11e-2005 Part 11: WLAN MAC and PHY specifications: MAC enhancements for Qos. Amendment IEEE Std. 802.11, 1999 Edition, 2005.

[2] ITU-T Recommendation G.1030 (2005) Estimating end-to-end performance in IP networks for data applications.

[3] M. Lora Girón. A Proposal for QoE in Cooperative Wireless Local Area Networks. In Proceedings of the Mobile Collaboration Systems Workshop at COOP 2010, Aix-en-Provence, France, 2010. 